\title{
Erratum to: Temperature-resistant and flexible supercapacitors based on 10-inch wafer-scale nanocarbon films (vol 62, pg 947, 2019)
}

Xiaobei Zang ${ }^{1^{*}}$, Yi Hou ${ }^{2}$, Teng Wang ${ }^{1}$, Rujing Zhang ${ }^{2}$, Feiyu Kang ${ }^{3^{*}}$ and Hongwei Zhu ${ }^{2 *}$

In the version of the article originally published in the volume 62, issue 7, 2019 of Sci China Mater (2019, 62 (7): $947-$ 954, https://doi.org/10.1007/s40843-018-9399-3), the authors used a wrong figure (Fig. 1) by mistake. The corrected version of Fig. 1 appears below.
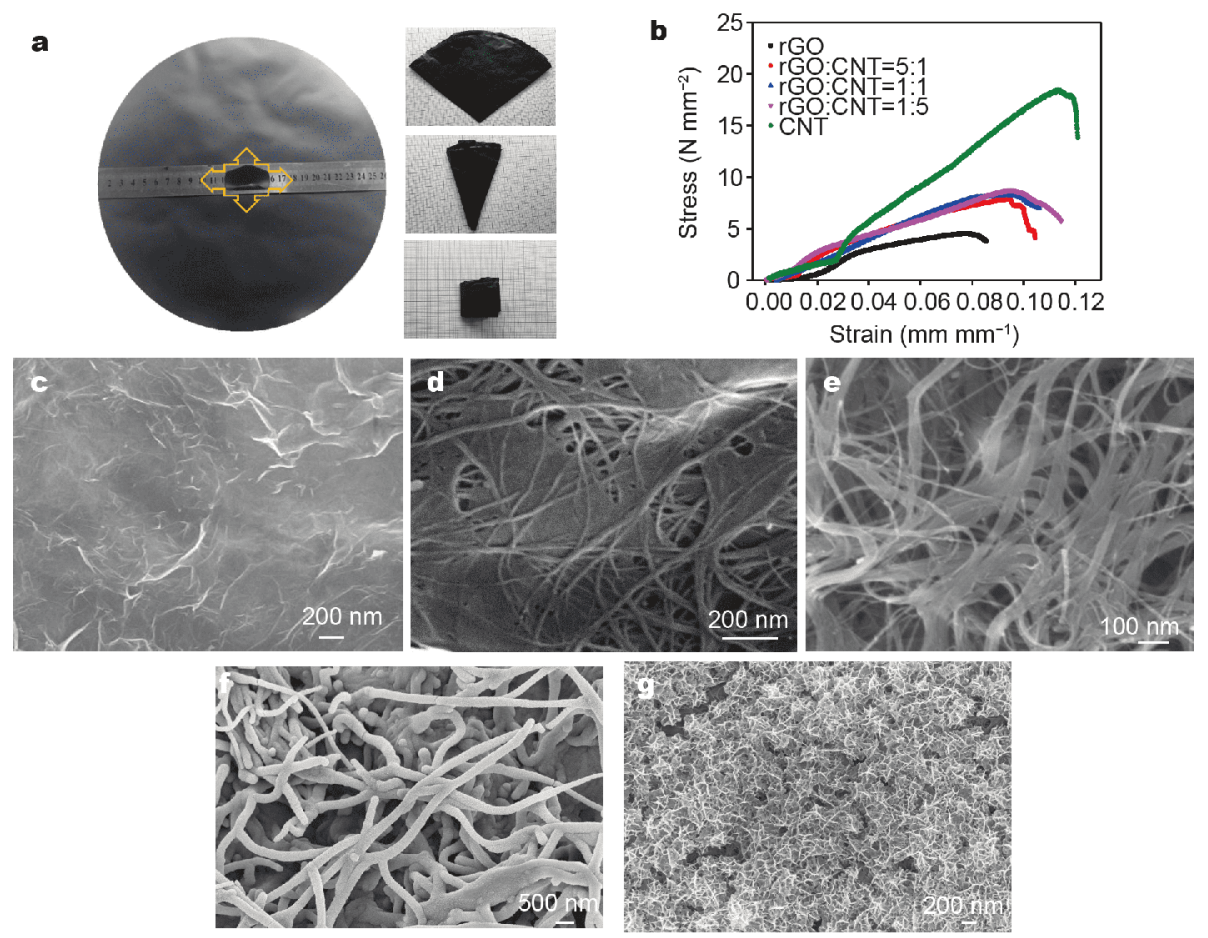

Figure 1 (a) Photographs, (b) tensile strain-stress curves and (c-e) SEM images of large-scale robust nanocarbon films, (f) SEM image, (g) highmagnification SEM image of $\mathrm{rGO} / \mathrm{CNT} / \mathrm{MnO}_{x}$ film.

\footnotetext{
${ }^{1}$ School of Materials Science and Engineering, China University of Petroleum (East China), Qingdao 266580, China

${ }^{2}$ State Key Laboratory of New Ceramics and Fine Processing, Center for Nano and Micro Mechanics (CNMM), School of Materials Science and Engineering, Tsinghua University, Beijing 100084, China

${ }^{3}$ Division of Energy and Environment, Graduate School of Shenzhen, Tsinghua University, Shenzhen 518055, China

* Corresponding authors (emails: xiaobeizang@upc.edu.cn (Zang X); fykang@tsinghua.edu.cn (Kang F); hongweizhu@tsinghua.edu.cn (Zhu H))
} 